Anna M. Mandich. Insegnare il francese in Italia. Repertorio di manuali pubblicati in epoca fascista (1923-1943).

Bologna : Cooperativa Libraria Universitaria Editrice Bologna, 2002 (Heuresis 3 Strumenti/Collana del Dipartimento di Lingue e Letterature Straniere Moderne 25), $201 \mathrm{p}$.

Francesco Paolo Alexandre Madonia

\title{
OpenEdition
}

Édition électronique

URL : https://journals.openedition.org/dhfles/1282

DOI : $10.4000 /$ dhfles. 1282

ISSN : 2221-4038

Éditeur

Société Internationale pour l'Histoire du Français Langue Étrangère ou Seconde

Édition imprimée

Date de publication : 1 juin 2004

Pagination : 181-183

ISSN : 0992-7654

Référence électronique

Francesco Paolo Alexandre Madonia, « Anna M. Mandich. Insegnare il francese in Italia. Repertorio di manuali pubblicati in epoca fascista (1923-1943). », Documents pour l'histoire du français langue étrangère ou seconde [En ligne], 32 | 2004, mis en ligne le 01 janvier 2012, consulté le 27 mai 2021. URL : http:// journals.openedition.org/dhfles/1282 ; DOI : https://doi.org/10.4000/dhfles.1282

Ce document a été généré automatiquement le 27 mai 2021.

(c) SIHFLES 


\section{Anna M. Mandich. Insegnare il francese in Italia. Repertorio di manuali pubblicati in epoca fascista (1923-1943).}

Bologna : Cooperativa Libraria Universitaria Editrice Bologna, 2002 (Heuresis 3 Strumenti/Collana del Dipartimento di Lingue e Letterature Straniere Moderne 25), $201 \mathrm{p}$.

Francesco Paolo Alexandre Madonia

1 Cet important volume d'Anna M. Mandich constitue la suite du Repertorio analitico dei manuali pubblicati dal 1625 al 1860 publié par Nadia Minerva et Carla Pellandra et du Repertorio 1860-1923, paru récemment sous la direction de Nadia Minerva.

2 La période 1923-1943 examinée est une des plus troublées de l'histoire italienne, mais aussi une des plus intéressantes du point de vue de l'histoire de la didactique à cause de la réforme scolaire promue par le philosophe et ministre de l'Instruction Publique de l'époque Giovanni Gentile.

3 Le répertoire proprement dit est composé de deux parties : la première présente une liste alphabétique de tous les manuels publiés pendant la période considérée; la deuxième présente un tableau chronologique détaillé et utile. La masse des données offertes est imposante : 748 titres de 283 auteurs différents. Pour constituer ce corpus Anna M. Mandich s'est livrée à de longues recherches d'archives, rendues difficiles du fait que certaines maisons d'édition n'ont plus aucune trace de leurs publications relatives à l'époque fasciste. La base pour recueillir les données lui a été fourniepar le Bollettino delle pubblicazioni italiane ricevute per diritto di stampa, publié à Florence par la Bibliothèque Nationale, mais aussi, pour les confrontations et les intégrations, par le Catalogo cumulativo del Bollettino (il CUBI). Le Bollettino delle pubblicazioni italiane est aussi la source utilisée par Anna M. Mandich pour établir la section du répertoire relative aux manuels pour l'enseignement des langues anglaise, allemande, espagnole, slovène, arabe, russe et hongroise; les données proposées ici au lecteur constituent, dans les intentions de l'Auteur, un point de départ pour un travail de comparaison entre l'enseignement des différentes langues étrangères en Italie à l'époque considérée. On 
doit tous souhaiter que les spécialistes de ces langues puissent dans les meilleurs délais offrir à la communauté scientifique des instruments de recherche analogues à ceux élaborés pour le français.

4 Un recueil de données a besoin pour être parfaitement compris d'un guide qui les organise et les élabore. Ce motif a probablement déterminé Anna M. Mandich à faire précéder son répertoire par deux essais, enrichis d'une vaste bibliographie et ayant pour objet, respectivement, L'insegnamento della lingua francese in epoca fascista (1923-1943) et Maschile e femminile nelle grammatiche del ventennio fascista. Le premier essai présente les résultats globaux de l'étude. Les manuels et les programmes sont pris en compte pour témoigner des progrès de la diffusion de l'idéologie fasciste dans tous les domaines des activités scolaires. Les manuels de français, qui n'échappent pas à ces dangereuses révisions, deviennent un véhicule de propagande du régime, comme tant d'autres. Le deuxième essai se propose d'analyser l'idée de 'masculin' et de 'féminin' dans les manuels pour l'enseignement du français, mais l'enquête menée par Anna M. Mandich est en fait de plus ample portée, car les deux tiers du chapitre se configurent plutôt comme une fresque vigoureuse du rôle de la femme selon les préceptes fascistes. Les exemples de version française fournis à la fin du chapitre constituent un frappant témoignage tant de la forte division des sexes que de l'effort de colporter le message fasciste par n'importe quel biais. La lecture des deux essais, qui avaient déjà été publiés, se révèle indispensable pour s'orienter dans la complexe politique de l'époque et pour en saisir les enjeux.

5 Le volume se signale à l'attention du lecteur par sa précision, sa clarté d'exposition, la richesse des références bibliographiques et la pertinence de l'argumentation de l'Auteur. Anna M. Mandich nous donne avec cet ouvrage un outil indispensable qui sera apprécié aussi bien par les spécialistes dans l'histoire de la didactique du français que par les historiens du fascisme qui y trouveront d'intéressantes remarques concernant l'histoire de la culture italienne.

\section{AUTEUR}

\section{FRANCESCO PAOLO ALEXANDRE MADONIA}

Università di Palermo 